\title{
Donor-Specific Anti-Human Leukocyte Antigen Antibodies Predict Prolonged Isolated Thrombocytopenia and Inferior Outcomes of Haploidentical Hematopoietic Stem Cell Transplantation
}

\author{
Xiaosu Zhao, ${ }^{1,2}$ Xiangyu Zhao, ${ }^{1,2}$ Mingrui Huo, ${ }^{1}$ Qiaozhen Fan, ${ }^{1}$ Xuying Pei, ${ }^{1}$ Yu Wang, ${ }^{1,2}$ \\ Xiaohui Zhang, ${ }^{1}$ Lanping $\mathrm{Xu},{ }^{1}$ Xiaojun Huang, ${ }^{1,2,3}$ Kaiyan Liu, ${ }^{1}$ and Yingjun Chang ${ }^{1,2}$ \\ ${ }^{1}$ Peking University People's Hospital and Peking University Institute of Hematology, Beijing Key Laboratory of Hematopoietic \\ Stem Cell Transplantation, No. 11 South Street of Xizhimen, Xicheng District, Beijing 100044, China \\ ${ }^{2}$ Collaborative Innovation Center of Hematology, Peking University, Beijing, China \\ ${ }^{3}$ Peking-Tsinghua Center for Life Sciences, Beijing 100871, China
}

Correspondence should be addressed to Yingjun Chang; rmcyj@bjmu.edu.cn

Received 11 October 2016; Revised 22 December 2016; Accepted 20 March 2017; Published 18 April 2017

Academic Editor: Mepur H. Ravindranath

Copyright ( 2017 Xiaosu Zhao et al. This is an open access article distributed under the Creative Commons Attribution License, which permits unrestricted use, distribution, and reproduction in any medium, provided the original work is properly cited.

\begin{abstract}
Prolonged isolated thrombocytopenia (PT) after allogeneic stem cell transplantation (allo-SCT) has a great impact on transplant outcome. In this study, we performed a retrospective analysis to investigate the association of donor-specific anti-human leukocyte antigen (HLA) antibodies (DSAs) with PT in 394 patients who underwent unmanipulated haploidentical blood and marrow transplantation (HBMT). For HLA antibody positive samples with a median fluorescent intensity $(\mathrm{MFI})>500$, DSAs were further examined. A total of 390 patients (99.0\%) achieved sustained myeloid engraftment. Of the 394 cases tested, 45 (11.4\%) were DSA positive. The cumulative incidence of PT in this cohort of patients was $9.9 \pm 1.5 \%$. The incidence of PT was higher in patients with a MFI $\geq 1000$ compared with those with a MFI $<1000(16.8 \pm 6.4 \%$ versus $7.4 \pm 1.4 \%, P=0.05)$. Multivariate analysis showed that the presence of DSAs (MFI $\geq 1000$ ) was correlated to PT (hazard ratio (HR) 3.262; 95\% confidence interval (CI), 1.339-7.946; $P=0.009$ ) and transplant-related mortality (HR 2.320; 95\% CI, 1.169-4.426; $P=0.044$ ). Our results, for the first time, suggest an association of DSAs with PT after unmanipulated HBMT. It would help screen out the suitable donor and guide intervention. This indicated that DSAs should be incorporated in the algorithm for unmanipulated HBMT.
\end{abstract}

\section{Introduction}

For patients with hematologic malignancies, allogeneic stem cell transplantation (allo-SCT) is a kind of curative treatment [1-3]. Recently, haploidentical SCT provides alternative treatment options for patients lacking human-leukocyte antigen- (HLA-) matched related or unrelated donors. However, prolonged isolated thrombocytopenia (PT), which is defined as the engraftment of all peripheral blood cell lines other than a platelet (PLT) count $\geq 20 \times 10^{9} / \mathrm{L}$ or dependence on PLT transfusions for more than 90 days after allo-SCT, has a great impact on transplant outcomes, especially in haploidentical
SCT settings. The incidence of PT is around 5 to $37 \%$ after transplantation [4-6]. In our center, we established an unmanipulated haploidentical blood and marrow transplantation (HBMT) protocol that has a lower incidence of graft failure compared to other haploidentical transplant modalities [7], but PT still significantly increases the risk of transplantrelated mortality (TRM) $[4-6,8]$. Although the impaired PLT production and accelerated peripheral destruction are known to be the major causes of PT [4-6], there still might be other undiscovered factors that remain to be clarified [9].

Donor-specific antibodies (DSAs) are the anti-human leukocyte antigen (HLA) antibodies that specifically respond 
to the mismatched antigen of donor [10-12]. Several researchers, including us, have confirmed the effects of DSAs on graft failure (GF), including graft rejection (GR) and poor graft function (PGF), in patients who underwent haploidentical SCT either with $\mathrm{T}$ cell depletion or with $\mathrm{T}$ cell replete [13-15]. However, there is no data on the relationship of DSAs with PT after haploidentical SCT. Here, we performed a retrospective analysis to investigate the association of DSAs with the occurrence of PT in patients who underwent unmanipulated HBMT.

\section{Materials and Methods}

2.1. Patients. The consecutive patients who received unmanipulated HBMT from March 2010 to March 2014 at Peking University Institute of Hematology were enrolled in this study. All cases underwent DSA examination and had the complete data of DSA before transplantation. The transplant protocol was approved by the Institutional Review Board of Peking University People's Hospital, and the IRB approval number is 2012-27. The clinical trial registration number is NCT01617473. All patients signed informed consent forms. This study was conducted in accordance with the Declaration of Helsinki. The characteristics of patients and donors were shown in Table 1.

2.2. Transplant Protocol. The unmanipulated HBMT was performed as previously described [16, 17]. Patients were conditioned with busulfan (BU, $0.8 \mathrm{mg} / \mathrm{kg}$ iv, q6h), cyclophosphamide (CTX, $1.8 \mathrm{~g} / \mathrm{m}^{2} / \mathrm{d}$ for 2 days), and antithymocyte globulin (rabbit ATG, Sang Stat, Lyon, France) $(2.5 \mathrm{mg} /$ $\mathrm{kg} / \mathrm{d}$ iv for 4 days) or total body irradiation (TBI, 7.7 Gy), CTX, and ATG. All patients received G-CSF-mobilized bone marrow (BM) and peripheral blood stem cell transfusion. Cyclosporine A, mycophenolate mofetil, and short-term methotrexate were used for prophylaxis of graft-versushost disease (GVHD).

2.3. Anti-HLA Antibody and DSA Examination. The patients and donors underwent HLA allele typing of at least the A, B, and DRB1 loci routinely. The examination was performed as previously [15]. In brief, patient plasma/serum was screened for class I and class II HLA antibodies with a LABScreen Mixed Kit (One Lambda, Canoga Park, CA, USA). The samples were incubated with mixed HLA class I- and class IIcoated microspheres for $30 \mathrm{~min}$ in the dark and then washed before being incubated with anti-human immunoglobulin G-conjugated fluorescein isothiocyanate as described above for the first incubation. Finally, the samples were examined by a Luminex 200 flow analyzer (Luminex, Austin, TX, USA), and the data were analyzed with the HLA Fusion 3.2 software (One Lambda). The MFI of anti-HLA antibodies was obtained using the formula: sample beads - negative control beads. The samples with a MFI $>500$ were further tested for the specificity of the antibody (DSA), using a LABScreen Single Antigen Kit (One Lambda).

2.4. Definitions of Engraftment. Neutrophil engraftment was defined as achieving an ANC more than $0.5 \times 10^{9} / \mathrm{L}$ for three consecutive days, and platelet recovery was defined as
TABLE 1: Patient and donor characteristics.

\begin{tabular}{|c|c|}
\hline Characteristics & $n=394$ \\
\hline Median age (range), years & $26(2-58)$ \\
\hline Male sex, $n(\%)$ & $231(58.6 \%)$ \\
\hline \multicolumn{2}{|l|}{ Diagnosis } \\
\hline AML, $n(\%)$ & $160(40.6 \%)$ \\
\hline ALL, $n(\%)$ & $133(33.8 \%)$ \\
\hline CML, $n(\%)$ & $22(5.6 \%)$ \\
\hline MDS, $n(\%)$ & $41(10.4 \%)$ \\
\hline Others, $n(\%)$ & $38(9.6 \%)$ \\
\hline Disease status, SR (\%) & $301(76.4 \%)$ \\
\hline \multicolumn{2}{|l|}{ Conditioning regimen } \\
\hline MA, $n(\%)$ & $394(100 \%)$ \\
\hline \multicolumn{2}{|c|}{ Number of HLA-A, B, DR mismatched, $n(\%)$} \\
\hline 0 & $3(0.8 \%)$ \\
\hline 1 & $22(5.6 \%)$ \\
\hline 2 & $87(22.1 \%)$ \\
\hline 3 & $282(71.6 \%)$ \\
\hline \multicolumn{2}{|l|}{ Donor-recipient sex match, $n(\%)$} \\
\hline Male-male & $149(37.8 \%)$ \\
\hline Male-female & $102(25.9 \%)$ \\
\hline Female-male & $84(21.3 \%)$ \\
\hline Female-female & $59(15 \%)$ \\
\hline \multicolumn{2}{|c|}{ Donor-recipient relationship, $n(\%)$} \\
\hline Father-child & $150(38.1 \%)$ \\
\hline Mother-child & $57(14.5 \%)$ \\
\hline Sibling-sibling & $117(29 . \%)$ \\
\hline Child-parent & $58(14.7 \%)$ \\
\hline Others & $12(3.0 \%)$ \\
\hline \multicolumn{2}{|l|}{ ABO matched, $n(\%)$} \\
\hline Matched & $224(56.9 \%)$ \\
\hline Major mismatched & $74(18.8 \%)$ \\
\hline Minor mismatched & $22(5.6 \%)$ \\
\hline Bidirect mismatched & $74(18.8 \%)$ \\
\hline \multicolumn{2}{|c|}{ Cell compositions in allografts, median (range) } \\
\hline Infused nuclear cells, $10^{8} / \mathrm{kg}$ & $8.23(1.78-23.69)$ \\
\hline Infused CD $34+$ cells, $10^{6} / \mathrm{kg}$ & $2.61(0.39-16.82)$ \\
\hline Infused lymphocytes, $10^{8} / \mathrm{kg}$ & $2.93(0.16-9.49)$ \\
\hline Infused CD $3+$ cells, $10^{8} / \mathrm{kg}$ & $2.0(0.1-5.93)$ \\
\hline Infused CD $4+$ cells, $10^{8} / \mathrm{kg}$ & $1.1(0.15-3.94)$ \\
\hline Infused CD $8+$ cells, $10^{8} / \mathrm{kg}$ & $0.69(0.05-2.47)$ \\
\hline Infused CD14+ cells, $10^{8} / \mathrm{kg}$ & $1.48(0.19-6.13)$ \\
\hline \multicolumn{2}{|l|}{ aGVHD } \\
\hline Grade 0-I aGVHD & $259(65.7 \%)$ \\
\hline Grade II-IV aGVHD & $135(34.3 \%)$ \\
\hline \multicolumn{2}{|l|}{ cGVHD } \\
\hline No cGVHD & $266(67.5 \%)$ \\
\hline cGVHD & $128(32.5 \%)$ \\
\hline
\end{tabular}


TABLE 2: The HLA-antibodies and DSA in different statuses of engraftment.

\begin{tabular}{lcccc}
\hline & Good engraftment & PT & PGF & GR \\
\hline With positive anti-HLA antibody $(n, \%)$ & $27 / 295(9.2 \%)$ & $6 / 32(18.8 \%)$ & $12 / 39(30.8 \%)$ & $4 / 4(100 \%)$ \\
Median DSA MFI & 3096 & 9374 & 4843 & 17214 \\
Range of DSA MFI & $504-12969$ & $1403-18950$ & $600-11736$ & $3793-19948$ \\
\hline
\end{tabular}

achieving a platelet count more than $20 \times 10^{9} / \mathrm{L}$ without platelet transfusions for seven consecutive days. Chimerism analysis was performed by DNA fingerprinting for shorttandem repeats in blood samples and/or chromosome fluorescence in situ hybridization of BM samples [18]. Full donor chimerism was defined as $\geq 95 \%$ leukocytes of donor origin in peripheral blood samples. Mixed chimerism was defined as more than 5\% but less than $95 \%$ leukocytes of donor origin.

Primary GF consists of GR and PGF. GR is the failure to engraft neutrophils by day +28 for three consecutive days and the absence of donor hematopoiesis. PGF was defined as the presence of three cytopenic counts (ANC $\leq 0.5 \times 10^{9} / \mathrm{L}$, platelet $\leq 20 \times 10^{9} / \mathrm{L}$, and hemoglobin (HGB) $\leq 80 \mathrm{~g} / \mathrm{L}$ ) beyond day +28 with a complete donor chimerism in the absence of severe GVHD or hematological relapse. PT was defined as the engraftment of all peripheral blood cell lines other than a PLT count $\geq 20 \times 10^{9} / \mathrm{L}$ or dependence on PLT transfusions for more than 90 days after allo-HSCT in the presence of complete donor chimerism. Patients with evidence of PGF or hematologic relapse within 90 days after transplantation were excluded.

2.5. Statistical Analysis. The reference date of June 30th, 2016, was used to define the end of follow-up. The median follow-up was 796 (range: 25-2309) days. The Fisher exact test or Wilcoxon test was used for two-group comparisons. Disease-free survival (DFS), TRM, and overall survival (OS) were calculated according to Kaplan-Meier statistics. Death before $+90 \mathrm{~d}, \mathrm{GR}$, and PGF were considered a competing risk for PT. The difference of PT between groups was tested according to Gray's method, using R software for statistical computing. A two-sided $P$ value of 0.05 was considered significant. The log-rank test was used for comparisons of Kaplan-Meier curves. Potential prognostic factors for PT, OS, DFS, relapse, and TRM were examined using Cox proportional hazards models. SPSS 22.0 software was used (Mathsoft, Seattle, WA, USA) for statistical analysis.

\section{Results}

3.1. The General Clinical Characteristics of Patients. Total of 394 patients were enrolled in this study. The median age of patients was 26 years (range, 2-58 years). All patients received a myeloablative conditioning regimen. The median dose of infused CD34 ${ }^{+}$cells was $2.61(0.39-16.82) \times 10^{6} / \mathrm{kg}$. Except for patient age $(P=0.020)$, cases with PT and those without PT had equivalent patient and donor characteristics (Table 1).

3.2. Engraftment. Three hundred ninety patients achieved myeloid engraftment except for 4 patients (1.0\%). The median time to neutrophil engraftment was 13 days (range, 8-27 days). Up to the follow-up, the incidence of platelet engraftment was $91.9 \%$ and the median time to platelet engraftment was 17 days (range, 6-265 days). 32 patients did not meet the criteria of platelet engraftment. Total of 4 patients developed GR and 39 patients (9.9\%) were PGF. Among the patients who met the criteria of PGF, 9 patients died before +90 days and 16 patients later met the criteria of PT. In patients who met the criteria of PT, 16 patients only met the criteria of PT but not PGF. Thus, the cumulative incidence of $\mathrm{PT}$ in this cohort of patients was $9.9 \pm 1.5 \%$.

3.3. HLA Antibodies and DSA. Among all the patients, there were 99 (25.1\%) with positive anti-HLA antibody, consisted of 48 males and 51 females. Of these positive patients, 63 (16.0\%) had antibodies against HLA class I antigens and 57 (14.5\%) had antibodies against HLA class II antigens. 31 (7.9\%) patients had HLA antibodies against both classes I and II. In all HLA antibody positive patients, 45 (45.5\%) had positive DSA. Of the 394 cases tested, 45 (11.4\%) were DSA positive. Because we previously showed that high antibody titers of DSAs (MFI $\geq 10,000)$ were correlated to primary GR $(P<0.001)$ and that low antibody titers of DSAs $(\mathrm{MFI} \geq 2000)$ were strongly associated with primary PGF $(P=0.005)$ [15], here, we analyzed the impacts of DSAs $(\mathrm{MFI} \geq 1000)$ and DSAs $(\mathrm{MFI} \geq 2000)$ on PT. Of the DSA positive patients, the MFI of 37 (9.4\%) patients were more than 1000 and the MFI of $31(7.9 \%)$ patients were more than 2000. The detailed information about HLA antibodies and DSA was shown in Table 2.

3.4. Relationship of DSAs with PT after Unmanipulated HBMT. The percentages of cases with positive anti-HLA antibody were $9.2 \%(27 / 295)$ in patients with good engraftment, $18.8 \%(6 / 32)$ in patients with PT, $30.8 \%(12 / 39)$ in patients with PGF, and $100 \%$ in patients with GR (Table 2). By using a competing risk analysis, the incidence of PT was higher in patient with a MFI $\geq 1000$ compared with those with a MFI $<1000(16.8 \pm 6.4 \%$ versus $7.4 \pm 1.4 \%, P=0.05)$. For the competing events including death before $+90 \mathrm{~d}$, GR, and PGF, the incidence of PT was higher in patients with a MFI $\geq 1000$ compared with those with a MFI $<1000$ (29.9 $\pm 7.7 \%$ versus $6.5 \pm 1.3 \%, P<0.001)$. Univariate analysis showed that factors including elder $(P=0.020)$, infused lower dose of CD34 ${ }^{+}$cells $(P=0.061)$, positive HLA antibody $(P=0.092)$, and HLA class II antibody $(P=0.056)$ and DSAs (MFI $\geq 1000, P=0.058$ ) were associated with PT after unmanipulated HBMT (Table 3). However, multivariate analysis showed that the presence of DSAs (MFI $\geq 1000)$ (hazard ratio (HR) 3.262; 95\% confidence interval (CI), 1.339-7.946; $P=0.009)$ was independently associated with PT. It also 
TABle 3: Patient characteristics in groups with and without PT.

\begin{tabular}{|c|c|c|c|}
\hline & $\mathrm{PT}$ & Without PT & $P$ value \\
\hline$N$ & 32 & 362 & \\
\hline Patient age, median (range) & $36(3-54)$ & $26(2-58)$ & 0.020 \\
\hline Patient sex, male, $n(\%)$ & $17(53.1)$ & $214(59.1)$ & 0.320 \\
\hline Diagnosis, $n(\%)$ & & & 0.510 \\
\hline AML & $13(40.6)$ & $147(40.6)$ & \\
\hline ALL & $14(43.8)$ & $119(32.9)$ & \\
\hline CML & $1(3.1)$ & $21(5.8)$ & \\
\hline MDS & $3(9.4)$ & $38(10.5)$ & \\
\hline Others & $1(3.1)$ & $37(10.2)$ & \\
\hline \multicolumn{4}{|l|}{ Disease risk, $n(\%)$} \\
\hline SR & $22(68.8)$ & $279(77.1)$ & 0.288 \\
\hline $\mathrm{HR}$ & $10(31.3)$ & $83(22.9)$ & \\
\hline HLA incompatibility, $n(\%)$ & & & 0.533 \\
\hline 0 locus & 0 & $3(0.8)$ & \\
\hline 1 locus & $2(6.3)$ & $20(5.5)$ & \\
\hline 2 loci & $4(12.5)$ & $83(22.9)$ & \\
\hline 3 loci & $26(81.3)$ & $256(70.7)$ & \\
\hline Donor-patient relation, $n(\%)$ & & & 0.260 \\
\hline Sibling donor & $9(28.1)$ & $108(29.8)$ & \\
\hline Father donor & $4(12.5)$ & $53(14.6)$ & \\
\hline Mother donor & $9(28.1)$ & $141(39.0)$ & \\
\hline Children donor & $9(28.1)$ & $49(13.5)$ & \\
\hline Others & $1(3.1)$ & $11(3.0)$ & \\
\hline Donor-patient sex match, number (\%) & & & 0.722 \\
\hline Male to male & $11(34.4)$ & $138(38.1)$ & \\
\hline Male to female & $11(34.4)$ & $91(25.1)$ & \\
\hline Female to male & $6(18.8)$ & $78(21.5)$ & \\
\hline Female to female & $4(12.5)$ & $55(15.2)$ & \\
\hline ABO matched, $n(\%)$ & & & 0.213 \\
\hline Matched & $15(46.9)$ & $209(57.7)$ & \\
\hline Major mismatched & $8(25.0)$ & $66(18.2)$ & \\
\hline Minor mismatched & $5(15.6)$ & $69(19.1)$ & \\
\hline Bidirect mismatched & $4(12.5)$ & $18(5.0)$ & \\
\hline \multicolumn{4}{|l|}{ Cell compositions in allografts, $n(\%)$} \\
\hline Infused nuclear cells $\geq$ median & $15(46.9)$ & $181(50.0)$ & 0.735 \\
\hline Infused CD $34^{+}$cells $\geq$median & $11(34.4)$ & $187(51.7)$ & 0.061 \\
\hline Infused lymphocytes $\geq$ median & $13(48.1)$ & $160(50.3)$ & 0.829 \\
\hline Infused $\mathrm{CD}^{+}$cells $\geq$median & $14(51.9)$ & $160(50.3)$ & 0.878 \\
\hline Infused $\mathrm{CD} 4^{+}$cells $\geq$median & $16(59.3)$ & $159(50.0)$ & 0.356 \\
\hline Infused $\mathrm{CD}^{+}$cells $\geq$median & $16(59.3)$ & $157(49.4)$ & 0.324 \\
\hline Infused CD $14^{+}$cells $\geq$median & $16(59.3)$ & $157(49.4)$ & 0.324 \\
\hline Grade II-IV aGVHD & $12(44.4)$ & $105(33.0)$ & 0.229 \\
\hline cGVHD & $8(25.0)$ & $120(33.2)$ & 0.320 \\
\hline HLA antibody positive & $12(37.5)$ & $87(24.0)$ & 0.092 \\
\hline HLA-I antibody positive & $6(18.8)$ & $57(17.9)$ & 0.579 \\
\hline HLA-II antibody positive & $8(25.0)$ & $49(13.5)$ & 0.056 \\
\hline HLA-DP antibody positive & $3(9.4)$ & $29(8.0)$ & 0.732 \\
\hline DSA positive & $6(18.8)$ & $39(10.8)$ & 0.174 \\
\hline
\end{tabular}


TABLe 3: Continued.

\begin{tabular}{lccc}
\hline & PT & Without PT & $P$ value \\
\hline MFI $>1000$ & $6(18.6)$ & $31(8.6)$ & $\mathbf{0 . 0 5 8}$ \\
MFI $>2000$ & $4(12.5)$ & $27(7.5)$ & 0.310 \\
\hline
\end{tabular}

AML: acute myeloid leukemia; ALL: acute lymphoblastic leukemia; CML: chronic myeloid leukemia; MDS: myelodysplastic syndrome; SR: standard risk; HR: high risk; aGVHD: acute graft-versus-host disease; cGVHD: chronic graft-versus-host disease; HLA-I/II: class I/II HLA antibody; HLA-DP: class I and II HLA antibody double positive; DSA: donor-specific antibody; DSA-DP: class I and II DSA double positive; MFI: median fluorescent intensity. Bold means variates included in the multivariate analysis since their $P$ value $<0.1$ in the univariate analysis.

TABLE 4: Multivariate analysis of factors associated with transplant outcomes.

\begin{tabular}{lccc}
\hline & HR & $95 \%$ CI & $P$ value \\
\hline $\begin{array}{l}\text { Prolonged isolated } \\
\text { thrombocytopenia } \\
\text { Infused CD34+ cells } \geq \text { median }\end{array}$ & 0.481 & $0.232-1.001$ & 0.050 \\
$\quad$ DSA & & & \\
$\quad$ MFI $\geq 1000$ & 3.262 & $1.339-7.946$ & 0.009 \\
TRM & & & \\
$\quad$ Disease status & 1.753 & $(0.985-3.118)$ & 0.056 \\
$\quad$ Infused CD4+ cells $\geq$ median & 1.899 & $(1.079-3.340)$ & 0.026 \\
$\quad$ DSA & & & \\
$\quad$ MFI $\geq 1000$ & 2.320 & $(1.169-4.426)$ & 0.044 \\
Relapse & & & \\
$\quad \begin{array}{l}\text { Disease status } \\
\text { HLA-DP }\end{array}$ & 3.882 & $(2.103-7.166)$ & $<0.001$ \\
DFS & 2.552 & $(1.139-5.742)$ & 0.0230 \\
$\quad \begin{array}{l}\text { Disease status } \\
\text { cGVHD }\end{array}$ & 2.667 & $(1.761-4.038)$ & $<0.001$ \\
OS & 0.622 & $(0.423-0.916)$ & $<0.016$ \\
Disease status & & & \\
cGVHD & 2.504 & $(1.643-3.817)$ & $<0.001$ \\
DSA & 0.612 & $(0.413-0.908)$ & 0.015 \\
$\quad$ MFI $\geq 1000$ & 1.220 & $(0.998-2.138)$ & 0.082 \\
\hline
\end{tabular}

seemed that an infused lower dose of $\mathrm{CD} 34^{+}$cells might predict a higher incidence of PT (HR 0.481; 95\% CI, 0.232$1.001 ; P=0.05$, Table 4).

3.5. Relationship of DSAs with Transplant Outcomes. To further investigate the relationship of DSAs with other transplant outcomes including TRM, relapse, DFS, and OS, we also took the above factors into the univariate and multivariate analyses. In the univariate analysis, DSAs (MFI $\geq 1000$, $P=0.076)$, grade II-IV acute GVHD $(P=0.033)$, infused $\mathrm{CD}^{+}(P=0.024)$ and $\mathrm{CD}^{+}$cells $(P=0.088)$, donorrecipient relationship $(P=0.097)$, and disease status at the transplantation $(P=0.029)$ were associated with TRM. The results of multivariate analysis showed that an infused higher dose of $\mathrm{CD} 4^{+}$cells and DSAs (MFI $\geq 1000$ ) were the independent risk factors of TRM (Table 4). For relapse, disease status $(P<0.001)$, sex match $(P=0.09)$, and HLA-DP $(P=0.024)$ were brought into multivariate analysis. Finally, patients in high risk and HLA-DP were related to leukemia relapse (Table 4). For DFS, chronic GVHD $(P=0.011)$, disease status
$(P<0.001)$, and sex match $(P=0.094)$ were conducted into multivariate analysis. As our expected, patients in high risk and with chronic GVHD were associated with DFS (Table 4). It showed that with chronic GVHD $(P=0.006)$, disease status $(P<0.001)$ and DSAs $(\mathrm{MFI} \geq 1000, P=0.09)$ were related to OS in univariate and multivariate analyses though DSAs $(M F I \geq 1000)$ did not show a statistical significance (Table 4).

3.6. Effects of PT on Transplant Outcomes. Compared to cases with PT, patients without PT had lower incidence of TRM $(16.3 \pm 0.02 \%$ versus $32.5 \pm 0.09 \%, P=0.043)$, similar incidence of relapse $(12.3 \pm 0.02 \%$ versus $14.4 \pm 0.07 \%$, $P=0.677)$, and higher probabilities of DFS $(74.7 \pm 0.02 \%$ versus $56.3 \pm 0.88 \%, P=0.046)$ and $\mathrm{OS}(75.2 \pm 0.02 \%$ versus $56.3 \pm 0.88 \%, P=0.047$ ) (Figure 1). Multivariate analysis showed that the onset of PT was independently associated with TRM (HR, 2.717; 95\% CI, 1.343-5.498; $P=0.005$ ). Besides, patients with PT seemed also to have a worse DFS (HR, 1.695; 95\% CI, 0.984-2.921; $P=0.057$ ) and OS (HR, 1.629; 95\% CI, 0.928-2.858; $P=0.089$ ).

\section{Discussion}

The association of DSAs with graft failure has been demonstrated by other researchers and us in either HLA-matched unrelated donor transplant, umbilical cord blood transplantation, or haploidentical transplantation [10-12, 15, 19]. In this study, we, for the first time, found an association of the presence of DSAs with PT after unmanipulated HBMT, indicating that DSAs may be involved in the pathogenesis of this complication. In addition, patients with the onset of PT-experienced inferior transplant outcomes provide further evidence suggesting that the presence of DSAs should be incorporated in the donor selection algorithm [20,21].

The definition of a threshold for DSAs, according to MFI, is a premise for analyzing the association of DSAs with PT after transplantation. In previous studies, several cutoff values, such as MFI $>500,1000,1500,2000$, and 5000, have been defined as DSA positive that was associated with graft failure in different transplant modalities with different conditioning regimens and GVHD prophylaxis $[10,11,14,15,22]$. In this study, the cutoff value of DSA MFI was 1000, which was associated with the onset of PT after unmanipulated HBMT. Our previous observation indicated that patients with DSA MFI $\geq 10000$ experienced graft rejection and cases with DSA MFI $\geq 2000$ experienced PGF. Our results suggest that high, intermediate, and low antibody titers of DSAs may lead to GR, PGF, and PT, respectively [15]. The fact that patients with PT experienced higher cumulative incidence of 


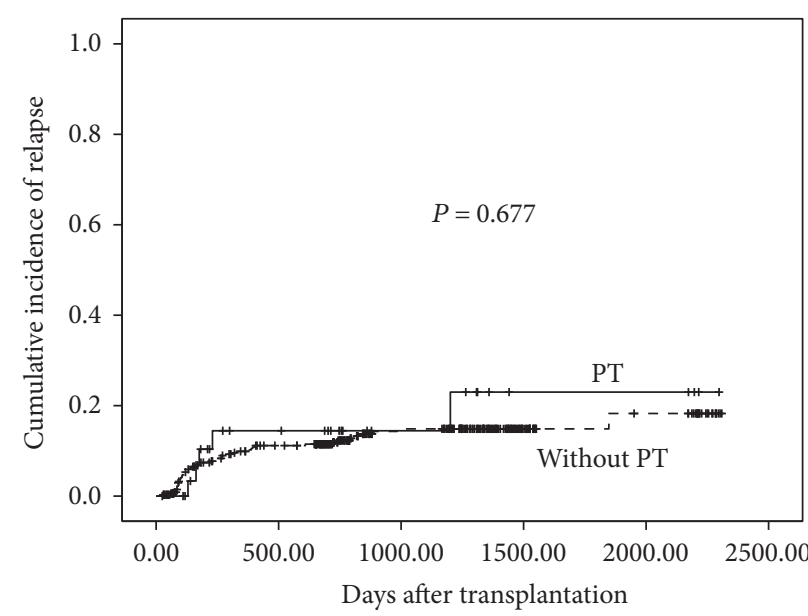

(a)

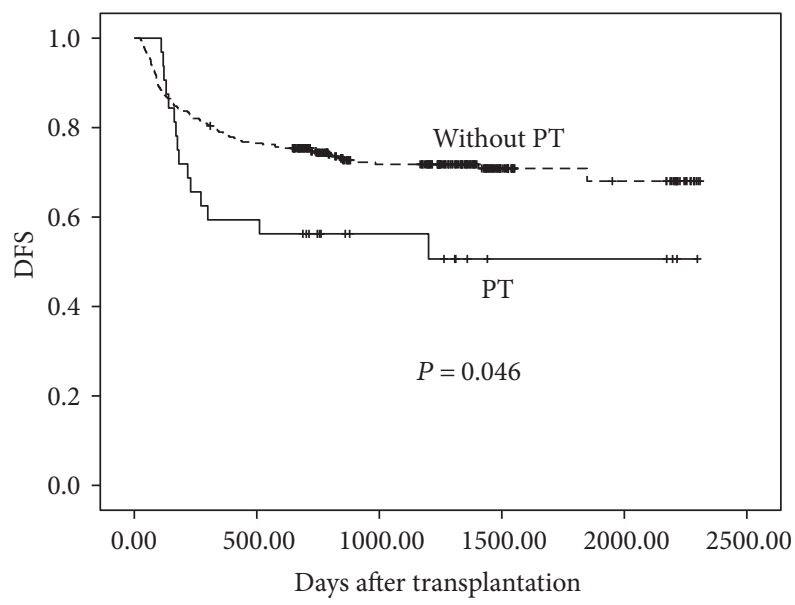

(c)

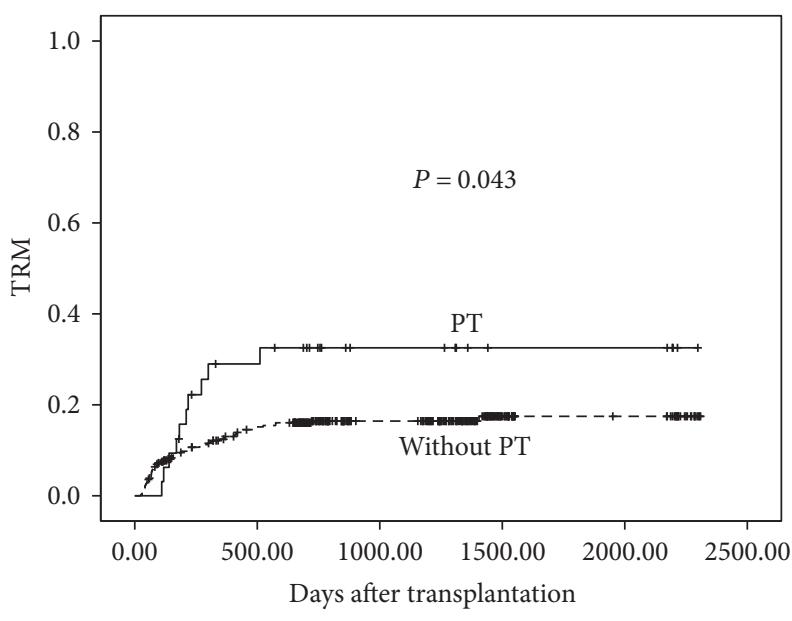

(b)

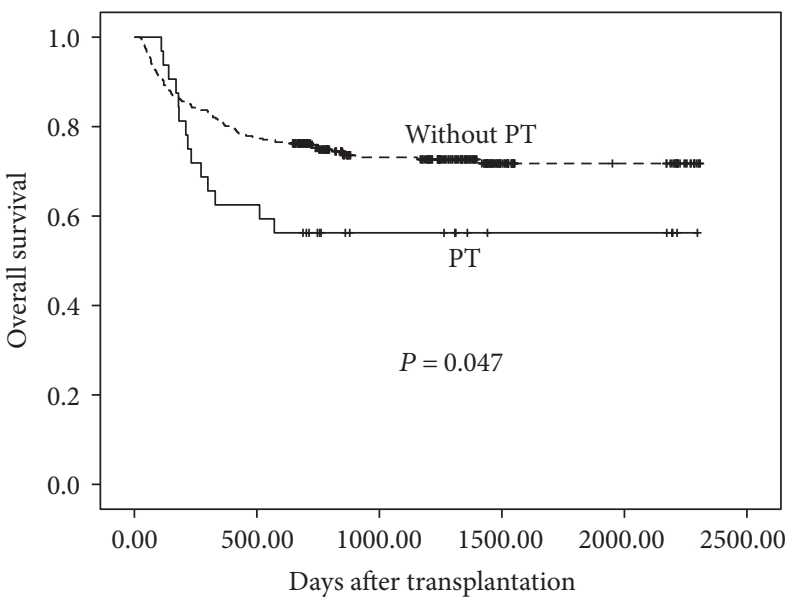

(d)

FIGURE 1: PT and transplant outcomes after haploidentical transplantation. (a) Cumulative incidence of relapse (CIR) and (b) TRM. (c) Disease-free survival. (d) Overall survival.

TRM and inferior DFS and OS supports the logical theory that the presence of DSAs results in PT, which may contribute to inferior survival.

Barge et al. [23] have found that DSAs may kill donor cells through antibody-dependent cell-mediated cytotoxicity (ADCC). In vitro experiment performed by us showed that DSAs could induce apoptosis of $\mathrm{CD} 34^{+}$cells and endothelial progenitor cells (EPCs) in the allografts (unpublished data). Kong et al. [24] from our center reported that the PT patients exhibited remarkable decreases in cellular elements of the vascular microenvironment of the bone marrow, including EPCs and perivascular cells, compared to the cases with good graft function after transplantation and the healthy donors, respectively. A multivariate analysis revealed that EPCs were an independent risk factor for PT. Their data suggested that an impaired $\mathrm{BM}$ vascular microenvironment may contribute to the occurrence of PT after allo-SCT [24]. It is reasonable that DSAs may lead to BM vascular microenvironment impairment via inducing apoptosis of EPCs, although further studies are needed. Our group also demonstrated that the recruitment of $\mathrm{CD}^{+} \mathrm{T}$ cells into BM might explain the suppression of megakaryocyte apoptosis through the elevated expression of
$\mathrm{CX}_{3} \mathrm{CR}^{+}{ }^{+}$in PT after allo-SCT [25]. These results indicate that an immune-mediated mechanism may contribute to the pathogenesis of PT after unmanipulated HBMT.

The present study had several limitations. First, our study is a single-center retrospective study. A prospective study with a training group and validation group is needed. Second, the present study only investigated the unmanipulated HBMT modality with an ATG conditioning regimen. Therefore, our results should be further confirmed in a multicenter clinical trial and in $\mathrm{T}$ cell-depleted haploidentical SCT modalities or in T cell-replete haploidentical SCT settings with postcyclophosphamide [26].

In conclusion, our results not only, for the first time, suggested an association of DSAs with PT after unmanipulated HBMT but also confirmed the effects of PT on inferior transplant outcomes. In addition, our study is clinically relevant and provides further evidence that DSAs should be incorporated in the algorithm for unmanipulated HBMT.

\section{Conflicts of Interest}

The authors declare no competing financial interests. 


\section{Authors' Contributions}

Yingjun Chang designed the study. Yingjun Chang and Xiaosu Zhao collected the data. Yingjun Chang and Xiaosu Zhao analyzed the data and wrote the manuscript. All authors contributed to the interpretation of the data, preparation of the manuscript, and approval of the final version.

\section{Acknowledgments}

The authors thank all the faculty members who participated in this study. This work was supported by the Milstein Medical Asian American Partnership Foundation, the Key Program of National Natural Science Foundation of China (81230013), the National Natural Science Foundation of China (81670175), the Health Science Promotion Project of Beijing (TG-2015-003), the Scientific Research Foundation for Capital Medicine Development (2016-1-4082), and Beijing Key Laboratory for Hematopoietic Stem Cell Transplantation.

\section{References}

[1] F. Aversa, A. Terenzi, A. Tabilio et al., "Full haplotypemismatched hematopoietic stem-cell transplantation: a phase II study in patients with acute leukemia at high risk of relapse," Journal of Clinical Oncology, vol. 23, no. 15, pp. 3447-3454, 2005.

[2] Y. J. Chang and X. J. Huang, "Haploidentical hematopoietic stem cell transplantation with unmanipulated granulocyte colony stimulating factor mobilized marrow and blood grafts," Current Opinion in Hematology, vol. 19, no. 6, pp. 454-461, 2012.

[3] Y. Reisner, D. Hagin, and M. F. Martelli, "Haploidentical hematopoietic transplantation: current status and future perspectives," Blood, vol. 118, no. 23, pp. 6006-6017, 2011.

[4] X. H. Zhang, S. Y. Zhou, R. Feng et al., "Increased prostacyclin levels inhibit the aggregation and activation of platelets via the $\mathrm{PI} 3 \mathrm{~K}-\mathrm{AKT}$ pathway in prolonged isolated thrombocytopenia after allogeneic hematopoietic stem cell transplantation," Thrombosis Research, vol. 139, pp. 1-9, 2016.

[5] X. H. Zhang, Q. M. Wang, J. M. Zhang et al., "Desialylation is associated with apoptosis and phagocytosis of platelets in patients with prolonged isolated thrombocytopenia after alloHSCT," Journal of Hematology \& Oncology, vol. 8, no. 1, p. 116, 2015.

[6] R. Yamazaki, M. Kuwana, T. Mori et al., "Prolonged thrombocytopenia after allogeneic hematopoietic stem cell transplantation: associations with impaired platelet production and increased platelet turnover," Bone Marrow Transplantation, vol. 38, no. 5, pp. 377-384, 2006.

[7] R. S. Mehta, R. M. Saliba, J. Chen et al., "Post-transplantation cyclophosphamide versus conventional graft-versus-host disease prophylaxis in mismatched unrelated donor haematopoietic cell transplantation," British Journal of Haematology, vol. 173, no. 3, pp. 444-455, 2016.

[8] Y. Kong, Y. Hu, X. H. Zhang et al., "Association between an impaired bone marrow vascular microenvironment and prolonged isolated thrombocytopenia after allogeneic hematopoietic stem cell transplantation," Biology of Blood and Marrow Transplantation, vol. 20, no. 8, pp. 1190-1197, 2014
[9] R. A. Nash, T. Gooley, C. Davis, and F. R. Appelbaum, "The problem of thrombocytopenia after hematopoietic stem cell transplantation," The Oncologist, vol. 1, no. 6, pp. 371-380, 1996.

[10] S. O. Ciurea, P. F. Thall, X. Wang et al., "Donor-specific antiHLA Abs and graft failure in matched unrelated donor hematopoietic stem cell transplantation," Blood, vol. 118, no. 22, pp. 5957-5964, 2011.

[11] C. Cutler, H. T. Kim, L. Sun et al., "Donor-specific anti-HLA antibodies predict outcome in double umbilical cord blood transplantation," Blood, vol. 118, no. 25, pp. 6691-6697, 2011.

[12] A. Ruggeri, V. Rocha, E. Masson et al., "Impact of donorspecific anti-HLA antibodies on graft failure and survival after reduced intensity conditioning-unrelated cord blood transplantation: a Eurocord, Societe Francophone d'Histocompatibilite et d'Immunogenetique (SFHI) and Societe Francaise de Greffe de Moelle et de Therapie Cellulaire (SFGM-TC) analysis," Haematologica, vol. 98, no. 7, pp. 1154-1160, 2013.

[13] S. Yoshihara, E. Maruya, K. Taniguchi et al., "Risk and prevention of graft failure in patients with preexisting donor-specific HLA antibodies undergoing unmanipulated haploidentical SCT," Bone Marrow Transplantation, vol. 47, no. 4, pp. 508-515, 2012.

[14] S. O. Ciurea, M. de Lima, P. Cano et al., "High risk of graft failure in patients with anti-HLA antibodies undergoing haploidentical stem-cell transplantation," Transplantation, vol. 88, no. 8, pp. 1019-1024, 2009.

[15] Y. J. Chang, X. Y. Zhao, L. P. Xu et al., "Donor-specific antihuman leukocyte antigen antibodies were associated with primary graft failure after unmanipulated haploidentical blood and marrow transplantation: a prospective study with randomly assigned training and validation sets," Journal of Hematology \& Oncology, vol. 8, no. 1, p. 84, 2015.

[16] Y. Wang, H. X. Fu, D. H. Liu et al., "Influence of two different doses of antithymocyte globulin in patients with standard-risk disease following haploidentical transplantation: a randomized trial," Bone Marrow Transplantation, vol. 49, no. 3, pp. 426-433, 2014.

[17] Y. Wang, D. H. Liu, K. Y. Liu et al., "Long-term follow-up of haploidentical hematopoietic stem cell transplantation without in vitro $\mathrm{T}$ cell depletion for the treatment of leukemia: nine years of experience at a single center," Cancer, vol. 119, no. 5, pp. 978-985, 2013.

[18] X. Y. Qin, G. X. Li, Y. Z. Qin et al., “Quantitative chimerism: an independent acute leukemia prognosis indicator following allogeneic hematopoietic SCT," Bone Marrow Transplantation, vol. 49, no. 10, pp. 1269-1277, 2014.

[19] S. Morin-Zorman, P. Loiseau, J. L. Taupin, and S. Caillat-Zucman, "Donor-specific anti-HLA antibodies in allogeneic hematopoietic stem cell transplantation," Frontiers in Immunology, vol. 7, p. 307, 2016.

[20] Y. J. Chang, L. Luznik, E. J. Fuchs, and X. J. Huang, "How do we choose the best donor for T-cell-replete, HLAhaploidentical transplantation?" Journal of Hematology \& Oncology, vol. 9, no. 1, p. 35, 2016.

[21] Y. Wang, Y. J. Chang, L. P. Xu et al., "Who is the best donor for a related HLA haplotype-mismatched transplant?" Blood, vol. 124 , no. 6 , pp. 843-850, 2014.

[22] S. Spellman, R. Bray, S. Rosen-Bronson et al., "The detection of donor-directed, HLA-specific alloantibodies in recipients of unrelated hematopoietic cell transplantation is predictive of graft failure," Blood, vol. 115, no. 13, pp. 2704-2708, 2010. 
[23] A. J. Barge, G. Johnson, R. Witherspoon, and B. Torok-Storb, "Antibody-mediated marrow failure after allogeneic bone marrow transplantation," Blood, vol. 74, no. 5, pp. 14771480, 1989.

[24] Y. Kong, Y. Hu, X. H. Zhang et al., "Association between an impaired bone marrow vascular microenvironment and prolonged isolated thrombocytopenia after allogeneic hematopoietic stem cell transplantation," Biology of Blood and Marrow Transplantation: Journal of the American Society for Blood and Marrow Transplantation, vol. 20, no. 8, pp. 1190-1197, 2014.

[25] X. H. Zhang, G. X. Wang, H. H. Zhu et al., "Recruitment of CD8(+) T cells into bone marrow might explain the suppression of megakaryocyte apoptosis through high expression of CX3CR1(+) in prolonged isolated thrombocytopenia after allogeneic hematopoietic stem cell transplantation," Annals of Hematology, vol. 94, no. 10, pp. 1689-1698, 2015.

[26] C. G. Kanakry, E. J. Fuchs, and L. Luznik, "Modern approaches to HLA-haploidentical blood or marrow transplantation," Nature Reviews. Clinical Oncology, vol. 13, no. 1, pp. 10-24, 2016. 


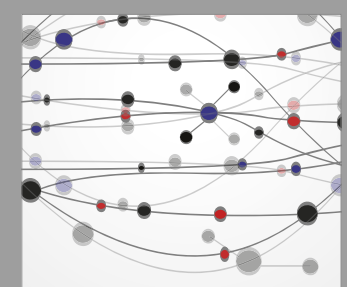

The Scientific World Journal
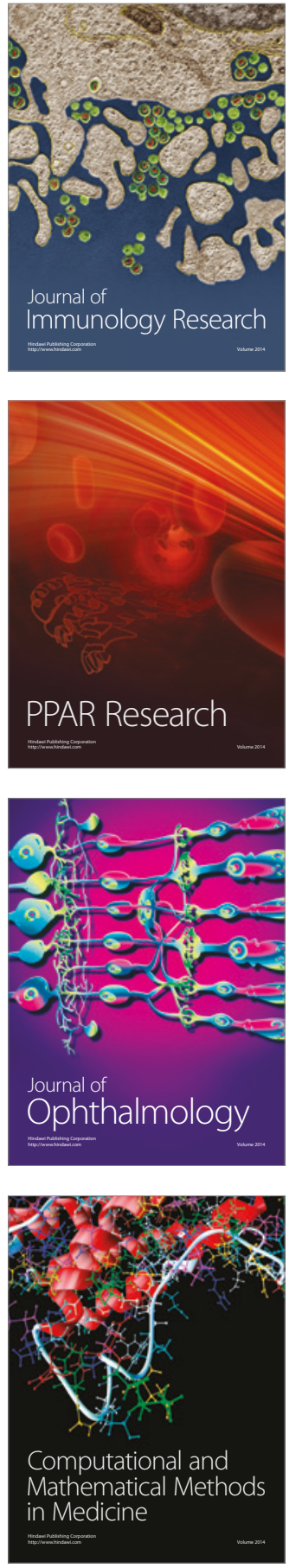

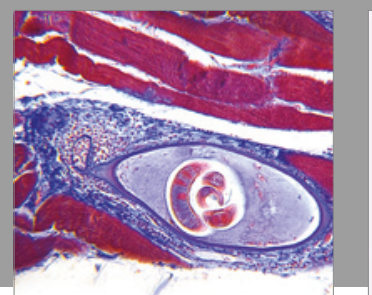

Gastroenterology Research and Practice
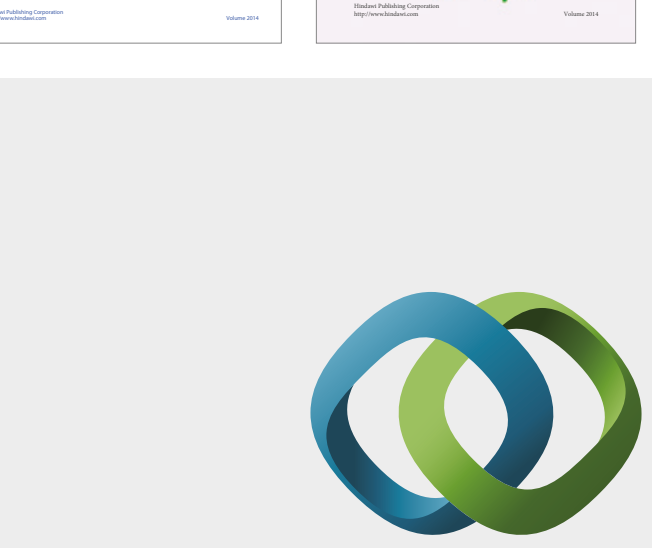

\section{Hindawi}

Submit your manuscripts at

https://www.hindawi.com
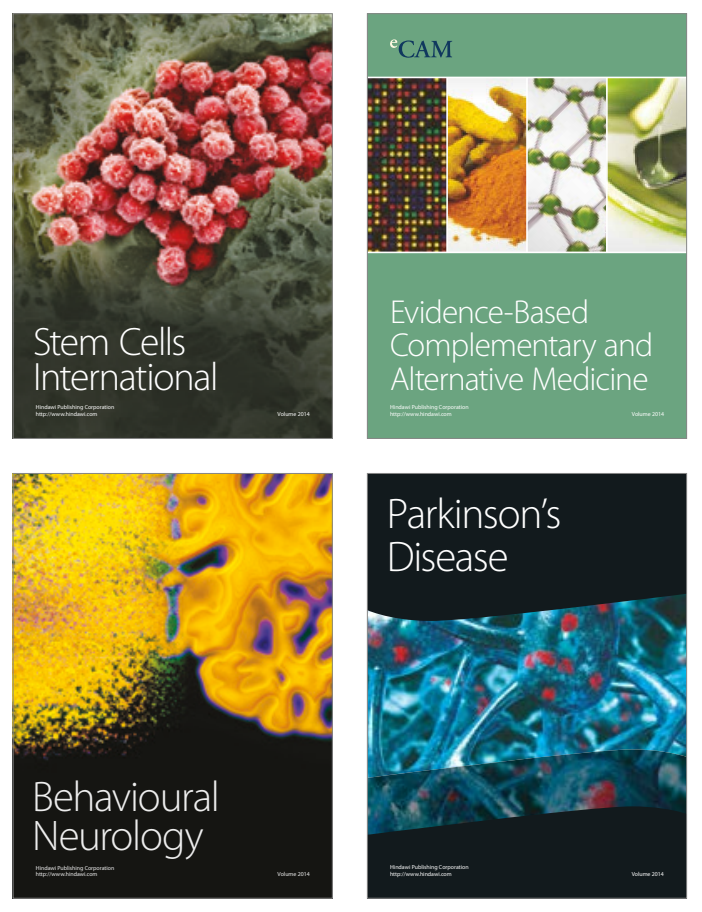
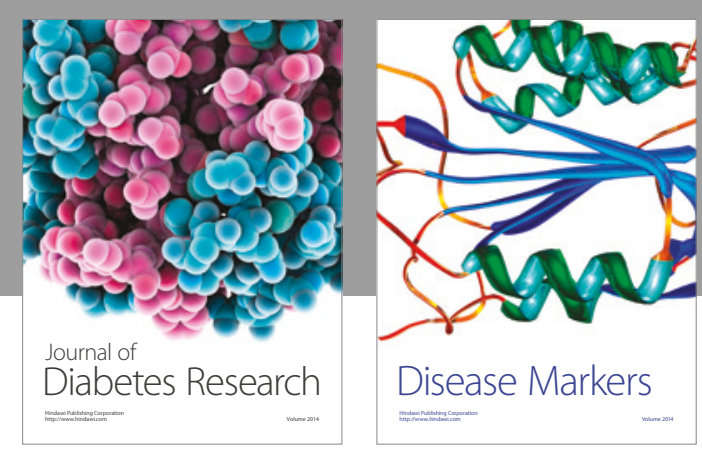

Disease Markers
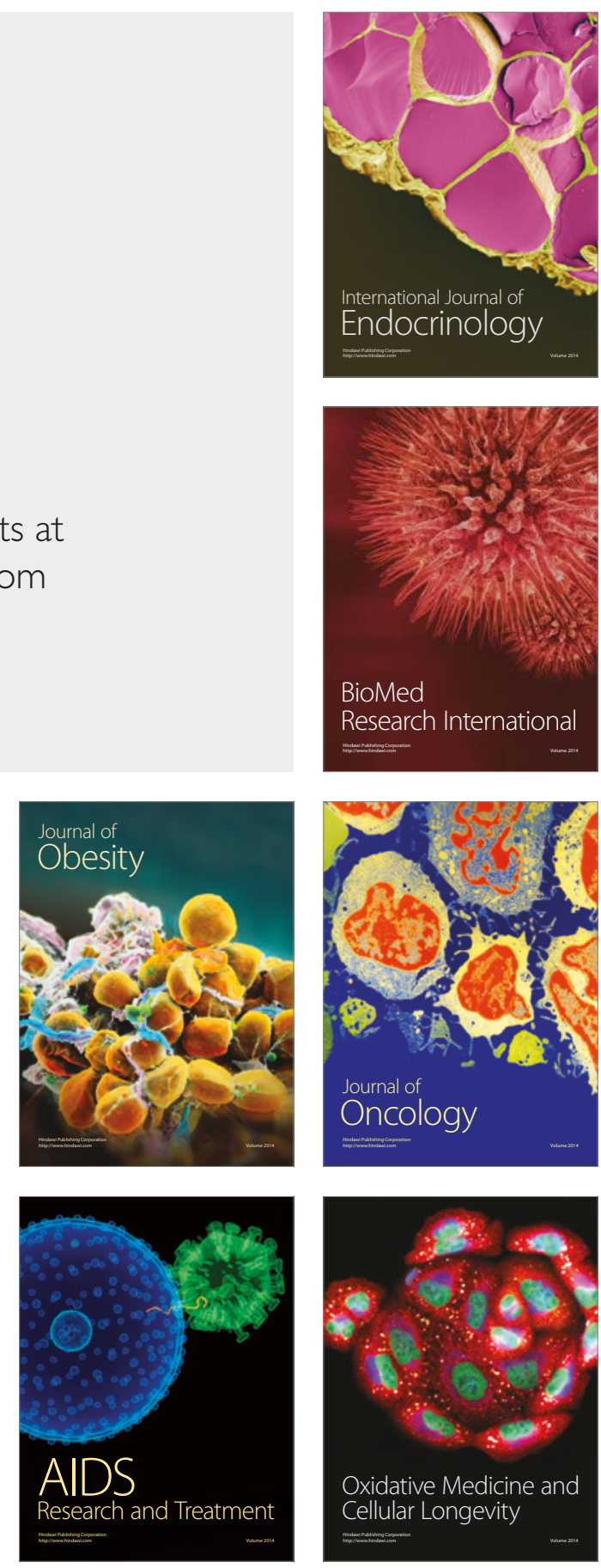